\title{
BRIGHTNESS CALIBRATION OF DIGITIZED OBJECTIVE-PRISM PLATES
}

\author{
D. ENGELS, L. CORDIS and T. KÖHLER \\ Sternwarte der Universität Hamburg \\ Gojenbergsweg 112 \\ D-21029 Hamburg \\ Germany
}

Currently, IIIa-J objective-prism plates from the Calar Alto and ESO Schmidt telescopes are used at the Hamburg Observatory to search for and to identify new astronomical objects. Major projects are the Hamburg Quasar Survey, the Hamburg-ESO Survey, and the Hamburg-Munich collaboration to identify sources from the ROSAT All-Sky Survey (cf. contributions to this conference by Hagen et al., Wisotzki, and Voges). The objective-prism plates are digitized with a PDS microphotometer. To determine the brightness of the digitized objects, a method to calibrate the plates was developed, using an internally determined characteristic curve and its external calibration with photometric sequences. Having spectral information, we are able to synthesize the Johnson B-filter as this filter is fully covered by the spectra.

The first step for the calibration of the plates is the construction of the characteristic curve over the pixels contributing to the flux in the B-filter. We assume that the curve is not wavelength dependent over this range. The characteristic curve is obtained from the density profiles perpendicular to dispersion, under the assumption of a point-spread-function (PSF) for the incident light. The basic parameters to describe the density profile are the peak-flux density $D_{\max }$ and the integral density $F$. The relation between the two is parametrized by a polygon, which is obtained by averaging the data (typically 20,000 spectra) in adequately chosen density intervals. Adopting a Moffat function for the PSF, this polygon determines the characteristic curve uniquely (Köhler 1991; Köhler et al. 1992).

The internally determined characteristic curves have to be calibrated with photometric standard stars. The characteristic curves of the IIIa-J plates depend on the batch, the hypersensitization, and the observing conditions. Therefore, each plate has to be calibrated individually using photometric sequences from the Guide Star Photometric Catalogue I (GSPC) for the bright end (B $<16 \mathrm{mag}$ ) and our own faint sequences $(15<\mathrm{B}<20 \mathrm{mag}$ ) obtained at the $1.2 \mathrm{~m}$ telescope on Calar Alto (Cordis et al. 1993, in preparation) for the faint end. Figure 1 shows an example of the capability of the method. For this particular plate we obtain a calibration with an error of $0.1 \mathrm{mag}$ (rms) over an 8 magnitude interval $(10.5<\mathrm{B}<18.5)$. Spectra down to B 19.5 are still recorded digitally; however at magnitudes $B>18.5$, the objects are close to the plate limit and the digitized data so noisy that neither a calibration not its evaluation is meaningful. Note that the spectra with B $<13$ mag are saturated and can be calibrated only after reconstruction of their true profiles (Köhler et al. 1992). This reconstruction is not always successful, so that on many plates the accurately calibrated range is $13<\mathrm{B}<18.5$. 
We performed tests with several hundred standard stars on about a dozen objective-prism plates to determine the limitations of the calibration method presented here. The main conclusions are:

1) we found no systematic differences between calibrations obtained from photometric sequences located in the corners and the centre of the same plate. This indicates that vignetting effects are smaller than the photometric accuracy achieved;

2) a photometric accuracy of $0.15 \mathrm{mag}$ (rms) can be achieved regularly over a magnitude interval of $13<B<18$. At fainter levels the errors increase rapidly. Down to $B=18.5$ the accuracy is $0.3 \mathrm{mag}(\mathrm{rms})$;

3) typical systematic errors of the order of $0.3 \mathrm{mag}$ are introduced if, in fields without faint photometric sequences, the calibration obtained from the GSPC-sequences is extrapolated to fainter magnitudes.

We conclude that the ability to calibrate the objective-prism plates homogeneously down to B $\sim 18.0$ allows the selection of flux-limited samples on the plates over a wide range of the sky. The photometric accuracy will be $0.15 \mathrm{mag}(\mathrm{rms})$, and even less if two plates of the same field are used. A high photometric precision is needed in particular for the study of the AGN population because its surface density increases strongly with fainter magnitudes. Due to photometric errors, we expect that the QSO surface density for B $<18$ mag coming from the Hamburg Quasar Survey has to be corrected by $10-15 \%$.

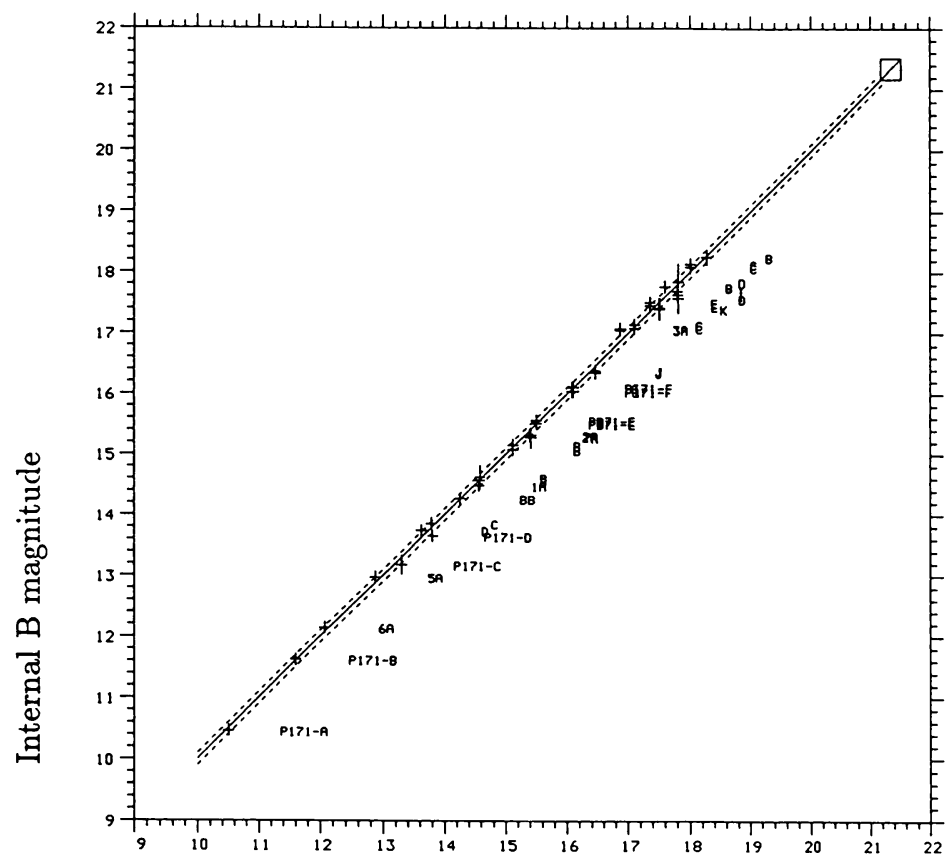

Photometric B magnitude

Figure 1. Calibration of the Calar Alto objective prism plate H1559 with photometric standard stars from the GSPC and CCD sequences in the Johnson B-band. The internal magnitudes were determined from an internal characteristic curve using several ten thousands of digitized spectra. 


\section{References}

Köhler, Th., 1991. Diploma Thesis, Hamburg University.

Kסhler, Th., Groote, D. and Wisotzki, L., 1992. 'Reconstruction of Saturated Spectra on Objective Prism Plates', in 4th ESO/ST-ECF Data Analysis Workshop, eds. P.J. Grosb $\varnothing 1$ and R.C.E. de Ruijsscher. 